\title{
The Antioxidants of Legume Nodule Mitochondria
}

\author{
Iñaki Iturbe-Ormaetxe, ${ }^{1}$ Manuel A. Matamoros, ${ }^{1}$ Maria C. Rubio, ${ }^{1}$ David A. Dalton, ${ }^{2}$ and \\ Manuel Becana ${ }^{1}$ \\ ${ }^{1}$ Departamento de Nutrición Vegetal, Estación Experimental de Aula Dei, CSIC, Apdo. 202, 50080 \\ Zaragoza, Spain; ${ }^{2}$ Biology Department, Reed College, Portland, OR 97202, U.S.A. \\ Submitted 9 May 2001; Accepted 14 June 2001.
}

\begin{abstract}
The mitochondria of legume root nodules are critical to sustain the energy-intensive process of nitrogen fixation. They also generate reactive oxygen species at high rates and thus require the protection of antioxidant enzymes and metabolites. We show here that highly purified mitochondria from bean nodules (Phaseolus vulgaris $\mathbf{L}$. cv. Contender $\times$ Rhizobium leguminosarum bv. phaseoli strain 3622) contain ascorbate peroxidase primarily in the inner membrane (with lesser amounts detected occasionally in the matrix), guaiacol peroxidases in the outer membrane and matrix, and manganese superoxide dismutase (MnSOD) and an ascorbate-regenerating system in the matrix. This regenerating system relies on homoglutathione (instead of glutathione) and pyridine nucleotides as electron donors and involves the enzymes monodehydroascorbate reductase, dehydroascorbate reductase, and homoglutathione reductase. Homoglutathione is synthesized in the cytosol and taken up by the mitochondria and bacteroids. Although bacteroids synthesize glutathione, it is not exported to the plant in significant amounts. We propose a model for the detoxification of peroxides in nodule mitochondria in which membrane-bound ascorbate peroxidase scavenges the peroxide formed by the electron transport chain using ascorbate provided by L-galactono1,4-lactone dehydrogenase in the inner membrane. The resulting monodehydroascorbate and dehydroascorbate can be recycled in the matrix or cytosol. In the matrix, the peroxides formed by oxidative reactions and by MnSOD may be scavenged by specific isozymes of guaiacol peroxidase, ascorbate peroxidase, and catalase.
\end{abstract}

Additional keywords: free radicals, nodule organelles, oxidative stress, thiols.

Reactive oxygen species (ROS), such as the superoxide radical and $\mathrm{H}_{2} \mathrm{O}_{2}$, are involved in all stages of legume nodule development, from initiation to senescence (Becana et al. 2000). In the nodule cytosol, ROS are generated mainly in oxidative reactions involving leghemoglobin and scavenged through the concerted action of copper-zinc superoxide dismutase (CuZnSOD) and of the four enzymes of the ascorbate-

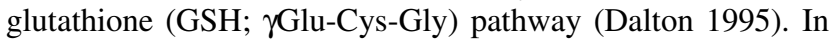
this pathway, ascorbate peroxidase (APX) catalyzes the reduction of $\mathrm{H}_{2} \mathrm{O}_{2}$ to water by ascorbate, and the resulting monode-

Corresponding author: M. Becana; Telephone: +34 976 716055; Fax: +34 976 716145; E-mail: becana@eead.csic.es hydroascorbate and dehydroascorbate are reduced back to ascorbate, respectively, by monodehydroascorbate reductase (MR), at the expense of NADH, and by dehydroascorbate reductase (DR) plus glutathione reductase (GR), at the expense of NADPH. Two key metabolites of this pathway are ascorbate and GSH, which are found at high concentrations in the cytosol (Becana et al. 2000; Dalton 1995). Some legume species and tissues, however, can synthesize another thiol tripeptide, homoglutathione (hGSH; $\gamma$ Glu-Cys- $\beta$ Ala), in place of GSH (Klapheck 1988; Matamoros et al. 1999). The role of hGSH in legume tissues is unclear but assumed to be similar to that of GSH (Bergmann and Rennenberg 1993).

The mitochondria are a main site for generation of ROS in the nodules because of the abundance of these organelles in the cortex and infected region (Millar et al. 1995b), their high rates of respiration required for active $\mathrm{N}_{2}$ fixation (Dalton 1995; Millar et al. 1995b), and their high content of heme and nonheme iron, which can catalyze the formation of hydroxyl radical from $\mathrm{H}_{2} \mathrm{O}_{2}$ (Becana et al. 2000). To prevent oxidative damage, nodule mitochondria should contain antioxidant enzymes and metabolites, but the antioxidant composition of nodule mitochondria is largely unknown. In a previous report on this subject, Dalton et al. (1993) could detect APX activity in mitochondria isolated from soybean nodules, but the enzyme was not recognized by the antibody raised to cytosolic APX. Thus, either there are two immunologically different APX enzymes in the cytosol and mitochondria, or the APX activity assayed in mitochondria corresponds to a secondary reaction of another enzyme such as a nonspecific guaiacol peroxidase (GPX). To provide insight into the mechanisms by which nodule mitochondria cope with ROS toxicity, it is essential to obtain virtually pure organelles, to assay antioxidant enzymes, and to determine their precise location within the mitochondria. This report describes the accomplishment of these objectives and discusses the compartmentation and physiological implications of strong antioxidant defenses in nodule mitochondria.

\section{RESULTS AND DISCUSSION}

Purification of nodule mitochondria.

Bean plants were thought to be ideal for this work because they produce the large amounts of nodules required for mitochondria purification and synthesize hGSH instead of GSH in the leaves and nodules (Matamoros et al. 1999). The bacteroids from bean nodules, like those from other legumes, only produce GSH (Moran et al. 2000), and thus an exchange of thiols between the bacteroid and the plant could be detected 
easily. Table 1 illustrates a typical purification of nodule mitochondria. The process involves differential centrifugation to obtain an organelle-enriched fraction and two sequential Percoll gradients. In the first gradient, three bands were evident, which correspond, respectively, to cellular debris and broken organelles, intact mitochondria (buoyant density of $1.065 \pm$ $0.001 \mathrm{~g}$ per $\mathrm{ml}$ ), and peroxisomes. In the second gradient, a single major band corresponding to intact mitochondria (buoyant density of $1.050 \pm 0.004 \mathrm{~g}$ per ml) was observed. Bands were identified on the basis of gradient fractionation and the assay of organelle markers.

The integrity of purified mitochondria was assessed by measuring the latency of the enzyme markers of the inner membrane (cytochrome $c$ oxidase) and matrix (malate dehydrogenase), as described below. Latency, calculated by comparing the activity of the enzymes in the presence ("broken organelles") and absence ("intact organelles") of Triton, revealed that the purified organelles were at least $90 \%$ intact. Additionally, the assay of organelle markers showed that the mitochondria had less than 5\% contamination with peroxisomes (uricase) and no detectable contamination with bacteroids (alanine dehydrogenase) or cytosol (leghemoglobin). The significantly higher residual catalase activity $(17.8 \%)$ compared with uricase activity (4.8\%), both typical enzymes of peroxisomes, could be ascribed to the presence of an additional catalase isozyme in nodule mitochondria, as reported for maize leaf mitochondria (Scandalios et al. 1980). Inhibitor studies suggest, however, that this activity was low, at best, and this aspect of the investigation was not pursued further.

\section{Peroxidases of nodule mitochondria.}

In contrast, a main thrust of this work was to demonstrate the presence in nodule mitochondria of genuine APX and related enzymes (and metabolites) and to localize them inside the mitochondria. To this purpose, it was first verified by fluorometry (Table 1) and immunological assay (data not shown) that mitochondria have no detectable contamination with leghemoglobin because this abundant cytosolic protein exhibits "pseudoperoxidase" activity with artificial substrates but not with ascorbate (Becana et al. 1989). Peroxidase activity was then assayed in mitochondria with ascorbate (APX activity) or pyrogallol (GPX activity) as substrates in the presence and absence of inhibitors (Table 2). Both activities were clearly detected and drastically inhibited by potassium cyanide $(\mathrm{KCN})$ or azide, as expected for hemoproteins. The use of $p$-chloromercuriphenyl sulfonic acid (pCMS) is critical to discriminate APX and GPX because this compound markedly inhibits APX but has little effect on typical GPX enzymes (Amako et al. 1994). Indeed, pCMS inhibited APX activity by $75 \%$, whereas it had no significant effect on the GPX activity

of nodule mitochondria. This suggests that mitochondrial APX, as its cytosolic and chloroplastic counterparts, contains thiol groups that are essential for catalytic activity or to maintain protein conformation (Chen and Asada 1989; Mittler and Zilinskas 1991). Another thiol reagent, $\beta$-mercaptoethanol, was found to inhibit APX and GPX activities to a similar extent. The GPX inhibition of nodule mitochondria by $\beta$-mercaptoethanol appears to contradict the inability of thiol reagents to inhibit typical leaf GPX activities (Chen and Asada 1989) but is fully consistent with the inhibition by dithiothreitol of GPX activity from maize leaf mitochondria (Prasad et al. 1995). Other compounds such as hydroxylamine, a suicide inhibitor of leaf APX (Chen and Asada 1990), and salicylhydroxamic acid, an inhibitor of alternative oxidase (Millar et al. 1995a), inhibited partially mitochondrial APX and GPX activities, whereas aminotriazole, a typical catalase inhibitor (Scandalios et al. 1980), had little effect (Table 2).

Another interesting observation of this work is that the mitochondrial APX does not lose its activity in the absence of ascorbate. Thus, we found similar APX activities when the mitochondria were purified with or without ascorbate supplementation in the extraction, isolation, and wash media. In this respect, the APX of nodule mitochondria behaves similarly to the cytosolic enzyme (Dalton 1995; Mittler and Zilinskas 1991) but contrarily to the enzymes of the chloroplasts (Amako et al. 1994), leaf peroxisomes (Jiménez et al. 1998), or potato tuber mitochondria (De Leonardis et al. 2000), which are inactivated by $\mathrm{H}_{2} \mathrm{O}_{2}$ in the absence of ascorbate. Interestingly, Ishikawa et al. (1998) reported the occurrence of a

Table 2. Inhibition of peroxidases from bean nodule mitochondria

\begin{tabular}{|c|c|c|}
\hline Inhibitor $^{\mathbf{a}}$ & $\begin{array}{c}\text { Ascorbate } \\
\text { peroxidase } \\
(\mathbf{A P X})^{\mathbf{b}}\end{array}$ & $\begin{array}{c}\text { Guaiacol } \\
\text { peroxidase } \\
(\mathbf{G P X})^{\mathbf{b}}\end{array}$ \\
\hline Control & $568 \pm 19(100)$ & $2,213 \pm 75(100)$ \\
\hline pCMS (0.5 mM) & $140 \pm 16(25)^{*}$ & $2,067 \pm 100(93)$ \\
\hline $\mathrm{KCN}(1 \mathrm{mM})$ & $156 \pm 10(27)^{*}$ & $25 \pm 15(1)^{*}$ \\
\hline $\mathrm{NaN}_{3}(1 \mathrm{mM})$ & $173 \pm 22(30)^{*}$ & $776 \pm 136(35)^{*}$ \\
\hline Hydroxylamine (2 mM) & $211 \pm 18(37)^{*}$ & $507 \pm 40(23)^{*}$ \\
\hline $\begin{array}{l}\text { Salicylhydroxamic acid } \\
\quad(0.5 \mathrm{mM})\end{array}$ & $302 \pm 20(53)^{*}$ & $1,137 \pm 28(51)^{*}$ \\
\hline 3-Amino-1,2,4-triazole (5 mM) & $504 \pm 22(89)$ & $1,651 \pm 96(75)^{*}$ \\
\hline$\beta$-Mercaptoethanol $(0.5 \mathrm{mM})$ & $204 \pm 21(36)^{*}$ & $823 \pm 52(37)^{*}$ \\
\hline \multicolumn{3}{|c|}{$\begin{array}{l}\text { a Mitochondrial extracts were assayed for APX and GPX activities in } \\
\text { the absence (control) or presence (with preincubation of } 8 \text { min) of each } \\
\text { inhibitor. } \\
\text { b Activities (nmol per min per mg of protein) are means } \pm \text { standard error } \\
\text { of the mean of four to six replicates, each corresponding to an inde- } \\
\text { pendent nodule extract. Residual activities are shown in parentheses. } \\
\text { Asterisk denotes a significant difference from control at } P=0.05 \\
\text { based on the Bonferroni-Dunnett's test. }\end{array}$} \\
\hline
\end{tabular}

Table 1. Purification of mitochondria from bean nodules

\begin{tabular}{lccc}
\hline Marker protein $^{\text {a }}$ & Nodule extract & Organelle-enriched pellet & Mitochondria (1st gradient) \\
\hline Cytochrome $c$ oxidase & $589 \pm 216$ & $2,483 \pm 211$ & $2,769 \pm 349$ \\
Uricase & $335 \pm 32$ & $130 \pm 9$ & $43 \pm 3$ \\
Catalase & $219 \pm 31$ & $197 \pm 15$ & $75 \pm 14$ \\
Alanine dehydrogenase & $77 \pm 6$ & 2 & 0 \\
Leghemoglobin & $25 \pm 3$ & 0 & 0 \\
\hline
\end{tabular}

a Data are means \pm standard error of the means of 3-6 samples, each corresponding to an independent nodule extract (gradient). Cytochrome $c$ oxidase, uricase, and alanine dehydrogenase activities are expressed in nmol per min per mg of protein, catalase activity in $\mu$ mol per min per mg of protein, and leghemoglobin content in nmol per mg of protein. 
membrane-bound APX in glyoxysomes that is not inactivated at least within the first $3 \mathrm{~h}$ of ascorbate depletion. These differences in enzyme stability may be due to the source of APX (organelle, tissue, and plant species) and, perhaps, to the existence of several APX isozymes in plant mitochondria (Jiménez et al. 1998).

Prasad et al. (1995) reported two other peroxidases in maize leaf mitochondria: a cytochrome $c$ peroxidase, localized in the membrane, and a GSH peroxidase, attributed to cytosolic contamination. The former activity was resistant to salicylhydroxamic acid, whereas the latter was resistant to hydroxylamine and, consequently, both peroxidase activities are distinct from the GPX activity we found in nodule mitochondria. Cytochrome $c$ peroxidase is generally regarded as being present in yeast mitochondria, but its occurrence in plants has not been established firmly (Dalton 1995). Its putative presence in maize leaf mitochondria (Prasad et al. 1995) is intriguing and, if this can be confirmed in other plant mitochondria, will have significant implications toward our understanding of peroxide metabolism in plants. Similarly, the role of GSH peroxidase, if any, in plant mitochondria remains unclear. Details for the assay of GSH peroxidase in mitochondria were not given (Prasad et al. 1995), but we could not detect such activity in whole nodule extracts (M. Becana and M. A. Matamoros, unpublished data) using strict controls (Smith and Shrift 1979). Part of this uncertainty might be due to the nonspecific affinities of peroxidases and to the difficulty of identifying the true in vivo reductant. For instance, GPX can utilize a wide range of reductants, but guaiacol (for which this enzyme is named) and pyrogallol are artificial compounds and the true reductant is elusive.

\section{Other antioxidants of nodule mitochondria.}

The three other enzymes involved in the ascorbate-GSH pathway of the chloroplasts and nodule cytosol (Dalton 1995) were also in bean nodule mitochondria (Table 3 ). Genuine DR, MR, and GR activities were detectable only when $\beta$-mercaptoethanol was included in the extraction medium and when careful controls such as boiled extracts were run in parallel to correct for nonenzymatic or oxidase activities. In addition,

Table 3. Specific activities and intramitochondrial localization of antioxidant enzymes ${ }^{\mathrm{a}}$

\begin{tabular}{lccc}
\hline Enzyme & Activity & Latency & Solubilization \\
\hline MnSOD & $42 \pm 1$ & $68 \pm 5$ & $85 \pm 8$ \\
APX & $552 \pm 21$ & 0 & $11 \pm 6$ \\
GPX & $2,473 \pm 205$ & $46 \pm 3$ & $48 \pm 6$ \\
MR & $104 \pm 9$ & $20 \pm 4$ & $75 \pm 1$ \\
DR & $26 \pm 1$ & $45 \pm 10$ & $82 \pm 1$ \\
hGR $^{\mathrm{b}}$ & $8 \pm 1$ & $53 \pm 3$ & $81 \pm 5$ \\
${\text { Cytochrome } c \text { oxidase }^{\mathrm{c}}}^{\mathrm{c}}$ & $3,984 \pm 189$ & $90 \pm 1$ & $3 \pm 1$ \\
Malate dehydrogenase $^{\mathrm{c}}$ & $63 \pm 3$ & $89 \pm 2$ & $77 \pm 1$ \\
\hline
\end{tabular}

${ }^{a}$ Manganese superoxide dismutase (MnSOD) activity is expressed in SOD units per $\mathrm{mg}$ of protein and malate dehydrogenase activity is expressed in $\mu \mathrm{mol}$ per min per mg. All other enzyme activities are expressed in nmol per min per mg protein. Latency and solubilization of enzymes are expressed in percent. Data are means \pm standard errors of the mean of 3-6 replicates, each corresponding to an independent nodule extract.

${ }^{\mathrm{b}}$ Homoglutathione reductase (hGR) activity was assayed with oxidized glutathione as substrate.

${ }^{c}$ Cytochrome $c$ oxidase and malate dehydrogenase were used as marker enzymes of the inner membrane and the matrix, respectively. nodule mitochondria showed high specific activity of SOD, which was identified as MnSOD on the basis of its insensitivity to $\mathrm{KCN}$ and $\mathrm{H}_{2} \mathrm{O}_{2}$ in mitochondrial crude extracts or in activity-stained gels. This agrees with the general observation that MnSOD is the major SOD isozyme in animal and plant mitochondria, including those from legume nodules (Becana et al. 1989; Puppo et al. 1987). However, we could not detect CuZnSOD or FeSOD on activity gels, despite the presence of both SOD isozymes in crude extracts of bean nodules (data not shown).

Because whole bean nodule extracts contain GSH and hGSH (Matamoros et al. 1999), we used high-performance liquid chromatography (HPLC) to analyze the thiol composition of bean nodule mitochondria. The analysis revealed that these organelles had no GSH but contained $13 \pm 2 \mathrm{nmol}$ of hGSH per mg of mitochondrial protein, a level that is comparable to the $20 \mathrm{nmol}$ of hGSH per mg of nodule protein for whole bean nodules (Matamoros et al. 1999). Therefore, the GR enzyme found in mitochondria should be functionally designated as a homoglutathione reductase (hGR). Because bean nodule mitochondria are devoid of the hGSH biosynthetic enzymes $\gamma$-glutamylcysteine synthetase and hGSH synthetase, whereas the latter activity is present in the nodule cytosol (Moran et al. 2000), it follows that the mitochondria obtain hGSH from the cytosol and utilize it as an antioxidant metabolite in place of GSH. A similar case may occur for the bacteroids, which also take up hGSH from the cytosol (Moran et al. 2000). The bacteroids, however, contain high levels of GSH as well as the corresponding biosynthetic enzymes $\gamma$-glutamylcysteine synthetase and GSH synthetase (Moran et al. 2000). To determine whether the exchange of thiol compounds between the bacteroids and the plant occurs in both directions, nodules were extracted, in parallel, under isosmotic and lysing conditions (Table 4). A sample of nodules was extracted isosmotically and the supernatant (cytosol) was acidified quickly to precipitate proteins, then neutralized and derivatized in the presence of dithioerythritol to preserve and quantify thiols. Another sample of nodules was extracted in acid conditions, the extract was sonicated and centrifuged, and thiols were quantified in the supernatant. This experiment revealed that the cytosol contained hGSH and a small amount of GSH (hGSH/GSH = 12), whereas the whole nodule extract contained high levels of GSH and hGSH (hGSH/GSH = 2). The latter values are similar to those reported (Matamoros et al. 1999) using acid extraction but no sonication, which indicates that methanesulfonic acid is sufficient to break the bacteroids.

Table 4. Thiol tripeptide composition of bean nodules

\begin{tabular}{lcc}
\hline Thiol $^{\text {a }}$ & Cytosol $^{\mathbf{b}}$ & Nodule extract $^{\mathbf{c}}$ \\
\hline Glutathione (GSH) & $18 \pm 4$ & $152 \pm 5$ \\
Homoglutathione (hGSH) & $215 \pm 17$ & $308 \pm 12$ \\
hGSH/GSH & 12 & 2 \\
\hline
\end{tabular}

a Thiol contents are expressed in nmol per $\mathrm{g}$ of fresh weight of nodules. Data are means \pm standard error of the mean of 3-6 independent nodule extracts.

${ }^{\mathrm{b}}$ Nodules were extracted in isosmotic conditions, and the extract was cleared by centrifugation. Supernatant (cytosol) was acidified immediately with methanesulfonic acid, centrifuged, and assayed for thiol content.

${ }^{\mathrm{c}}$ Nodules were extracted directly in methanesulfonic acid, the extract was centrifuged, and the supernatant was assayed for thiol content. 
Together these results show that the cytosol provides hGSH to the mitochondria and bacteroids, but also that bacteroids do not significantly export GSH to the cytosol. In fact, the bacteroids have substantial GR activity (Dalton et al. 1993), which probably reflects the importance of GSH (and hGSH) as a regulatory and antioxidant molecule for $\mathrm{N}_{2}$ fixation and other metabolic pathways of the bacteroids.

\section{Intramitochondrial localization of antioxidant enzymes.}

Cytochrome $c$ oxidase and malate dehydrogenase are located in the inner membrane and matrix of mitochondria, respectively. As indicated above, these two enzymes were used to assess the integrity of the outer and inner membranes of the purified mitochondria and also as controls for latency and solubilization studies of the antioxidant enzymes (Table 3). The latency of the enzymes was measured, as usual, by comparing activities in the presence and absence of Triton detergent. The solubilization of enzymes was measured by comparing activities in the pellet and supernatant after ultracentrifugation of the mitochondria that had been broken in liquid nitrogen. These studies showed conclusively that MnSOD, DR, and hGR are located in the matrix, whereas most APX is in the membrane. The zero latency of APX indicates that exogenous ascorbate is accessible directly to the enzyme, and therefore APX is located on the cytosolic side of the outer membrane or, assuming free permeation or quick transport of ascorbate through this membrane, on the outside of the inner membrane. To localize APX more precisely, mitochondria were subjected to controlled lysis and separated into outer membrane-enriched fraction, inner membrane-enriched fraction, and matrix, using antimycin A-insensitive cytochrome $c$ reductase, cytochrome $c$ oxidase, and malate dehydrogenase as the corresponding marker enzymes (Table 5). The activities of these marker enzymes reveal an approximately threefold enrichment in the outer or inner membranes and $<10 \%$ contamination of the matrix fraction with membranes. Data also show that most APX activity is associated with the inner membrane (Table 5). The APX activity in the outer membrane fraction is threefold lower than in the inner membrane fraction. This value is similar to that for cytochrome $c$ oxidase activity and thus the APX activity in the outer membrane fraction can be explained by contamination with inner membranes. An interesting and novel observation is that we occasionally detected APX activity in the matrix. The solubilization study showed that approximately $11 \%$ of the APX activity of mitochondria is in the matrix (Table 3), which corresponds to a specific activity

Table 5. Intramitochondrial localization of APX and GPX

\begin{tabular}{lccc}
\hline & $\begin{array}{c}\text { Outer } \\
\text { membrane- } \\
\text { enriched } \\
\text { fraction }\end{array}$ & $\begin{array}{c}\text { Inner } \\
\text { membrane- } \\
\text { enriched } \\
\text { fraction }\end{array}$ & Matrix \\
\hline Cytochrome $c$ oxidase & $2,043 \pm 650$ & $6,068 \pm 316$ & 0 \\
$\begin{array}{l}\text { Cytochrome } c \\
\quad \text { reductase }\end{array}$ & $265 \pm 15$ & $98 \pm 6$ & $30 \pm 3$ \\
$\begin{array}{l}\text { Malate dehydrogenase } \\
\text { APX }\end{array}$ & $7 \pm 1$ & $17 \pm 2$ & $172 \pm 38$ \\
GPX & $119 \pm 76$ & $369 \pm 29$ & $141 \pm 81$ \\
\hline
\end{tabular}

a Malate dehydrogenase activity is expressed in $\mu \mathrm{mol}$ per min per $\mathrm{mg}$ of protein. All other activities are expressed in nmol per min per $\mathrm{mg}$ of protein. Data are means \pm standard error of the mean of four independent mitochondrial extracts. of $141 \mathrm{nmol}$ per min per mg of protein (Table 5). This activity was fully abolished by pCMS, thus indicating the presence of genuine APX. Although this activity was not detected in all of our preparations, it seems clear that several APX isozymes may coexist in mitochondria. Thus, preliminary results of Jiménez et al. (1998) suggest that there are several APX isozymes in pea leaf mitochondria that can be resolved by isoelectric focusing. More recently, De Leonardis et al. (2000) purified and characterized APX from potato tuber mitochondria. Although no purity data were provided for the mitoplasts they isolated with digitonin and, hence, comparisons are not possible, De Leonardis and coworkers (2000) concluded that APX was inside the mitochondria, observations that are consistent with our localization of APX in the inner membrane and matrix.

Previous work on the presence of GPX in mitochondria has produced contradictory results. Prasad et al. (1995) detected membrane-bound GPX activity in maize leaf mitochondria, whereas Jiménez et al. (1997) did not find genuine GPX activity in pea leaf mitochondria. We found GPX activity in nodule mitochondria. In fact, solubilization data indicate that $48 \%$ of the activity is in the matrix and that there is significant activity associated with the membranes (Table 3). Fractionation studies of mitochondria confirmed the presence of GPX in both locations and indicate that bound GPX is predominantly in the outer membrane (Table 5). The activity of GPX in the inner membrane-enriched fraction can be accounted for by contamination with the outer membranes because antimycin A-insensitive cytochrome $c$ reductase and GPX activities were approximately threefold higher in the outer membrane fraction than in the inner membrane fraction. As expected, the GPX activity in the membrane or in the matrix was not inhibited by pCMS (an inhibitor of APX) or aminotriazole (an inhibitor of catalase). This indicates that the GPX activity we observed cannot be attributed to APX or catalase, two enzymes that exhibit GPX activity under certain conditions.

The relatively low latency of DR and hGR compared with other matrix enzymes (MnSOD and malate dehydrogenase), however, might be ascribed to the existence of transporters for the corresponding substrates dehydroascorbate and oxidized homoglutathione. Transporters for dehydroascorbate and ascorbate have been reported in various plant membranes, most notably the plasmalemma and chloroplast outer membrane (Horemans et al., 2000). Similarly, transporters for GSH were described in rat liver mitochondria (Lash 1995). It is uncertain, however, whether transporters for ascorbate, GSH, or their oxidized forms exist in plant mitochondria.

Several authors have reported recently on the presence of membrane-bound MR in leaf mitochondria and peroxisomes (Jiménez et al. 1997), as well as in spinach (Ishikawa et al. 1998) and oilseed (Bunkelmann and Trelease 1996) glyoxysomes. The data clearly show that most MR activity of nodule mitochondria is in the matrix because solubilization values for matrix-specific enzymes are typically 75 to $85 \%$ (Table 3 ). The relatively low latency of MR activity (20\%), however, suggests that the substrate monodehydroascorbate can reach the enzyme with relative ease. In contrast, the enzyme from pea leaf mitochondria was reported to have a latency of $89 \%$ and a solubility of $36 \%$ (Jiménez et al. 1997). Although we cannot provide an answer for the discrepancy in latency values, their solubility data are compatible, to some extent, with ours 
because it assumes the existence of membrane- and matrixspecific MR isozymes in various proportions, possibly depending upon the species, tissue, and growth stage. This is not surprising because MR is an ubiquitous enzyme in plant tissues, and multiple isozymes exist, for example, in the cell wall, cytosol, mitochondria, and probably other nodule organelles (Dalton et al. 1993).

In summary, we demonstrate in this work the existence, in bean nodule mitochondria, of two distinct peroxidases (APX in the membrane and GPX in the matrix and membrane) and a novel regenerating system for ascorbate which uses hGSH (instead of GSH) and pyridine nucleotides as electron donors. The results of enzyme localization, together with our previous work (Dalton et al. 1993; Moran et al. 2000), enabled us to propose a model for ROS metabolism in the mitochondria, cytosol, and bacteroids of legume nodules (Fig. 1). According to this model, the $\mathrm{H}_{2} \mathrm{O}_{2}$ that is formed directly (or derived from the superoxide radical) in the inner membrane by the electron transport chain is scavenged by membrane-bound APX. The ascorbate required for APX activity can be provided by the enzyme L-galactono-1,4-lactone dehydrogenase, which catalyzes the last step of ascorbate synthesis in the inner membrane of the mitochondria (Õba et al. 1995; Siendones et al. 1999). The resulting oxidized compounds, monodehydroascorbate and dehydroascorbate, can be recycled in the matrix or cytosol because both subcellular compartments contain MR, DR, and hGR. In the mitochondrial matrix, the $\mathrm{H}_{2} \mathrm{O}_{2}$ formed as a result of oxidative metabolism and of MnSOD activity could be scavenged directly by hGSH and other antioxidants, such as specific APX, GPX, and catalase isozymes. The thiol tripeptide hGSH found in mitochondria originates in the cytosol. Oxidized hGSH is recycled by hGR using NADPH, which can be formed from NADH by a mitochondrial transhydrogenase (Carlenor et al. 1988) or be taken up from the cytosol (Rasmusson and Møller 1990). The bacteroids can also import hGSH from the plant but do not export significant amounts of GSH to the cytosol, which indicates that hGSH functionally replaces GSH in the plant fraction of bean nodules and suggests that hGSH is important for both symbiotic partners.

\section{MATERIALS AND METHODS}

\section{Biological material.}

Nodulated bean plants (Phaseolus vulgaris L. cv. Contender $\times$ Rhizobium leguminosarum bv. phaseoli strain 3622) were grown in controlled environment chambers, as described else-

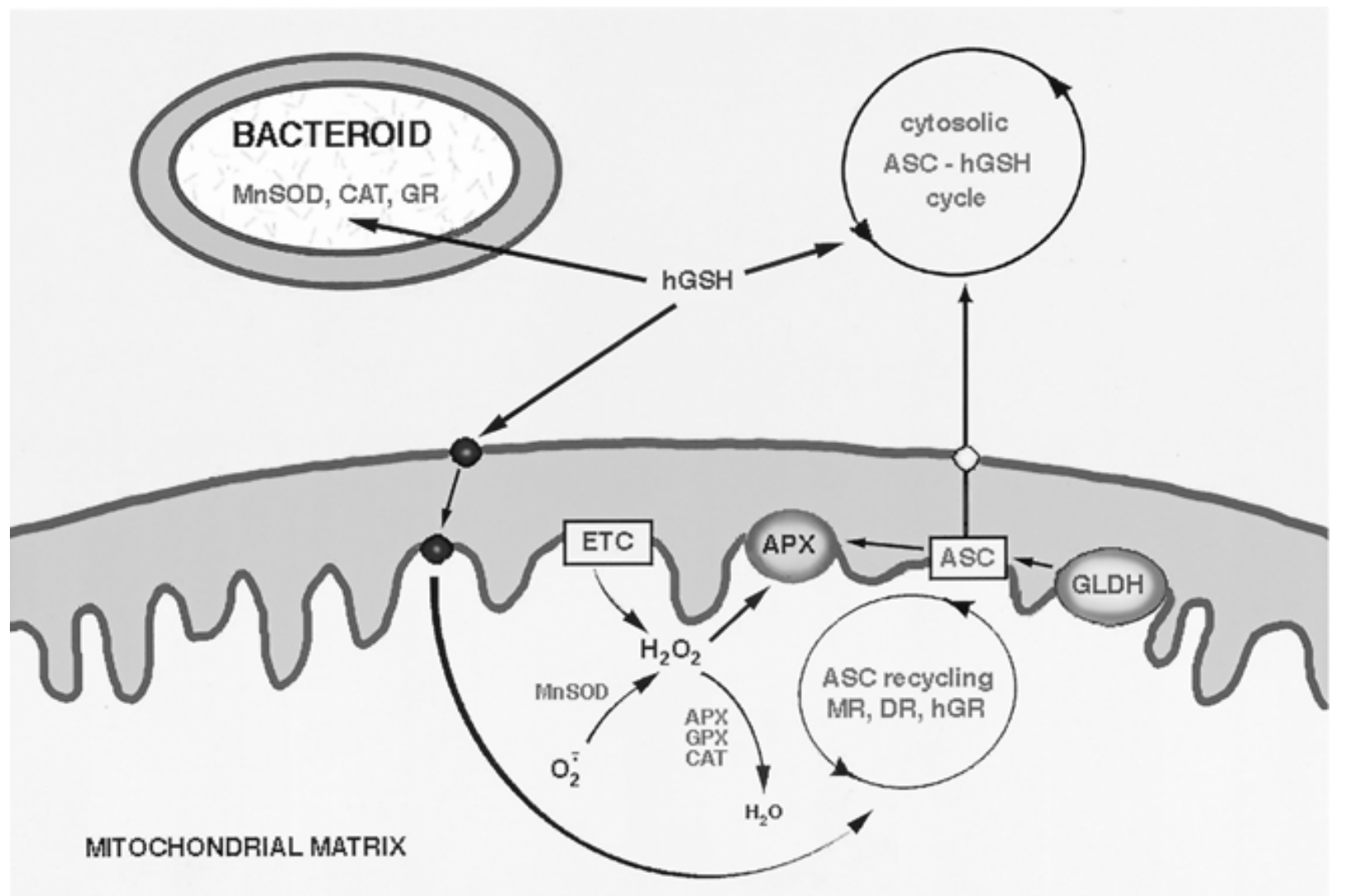

Fig. 1. Model of antioxidant processes in legume nodule mitochondria. The interaction with reactive oxygen species (ROS) metabolism in the bacteroids and cytosol is also shown. $\mathrm{H}_{2} \mathrm{O}_{2}$ generated in the electron transport chain (ETC) is scavenged by ascorbate peroxidase (APX) using ascorbate (ASC) provided by L-galactono-1,4-lactone dehydrogenase (GLDH). Both enzymes are located in the inner membrane. The resulting oxidized products, monodehydroascorbate and dehydroascorbate, can be reduced back to ASC in the matrix and cytosol by monodehydroascorbate reductase (MR), with NADH as the electron donor, or by dehydroascorbate reductase (DR) and homoglutathione reductase (hGR), with NADPH as the electron donor. ROS generated in the matrix as a result of oxidative metabolism and of manganese superoxide dismutase (MnSOD) activity can be destroyed directly by homoglutathione (hGSH) or by antioxidant enzymes, possibly including specific isozymes of APX, guaiacol peroxidase (GPX), and catalase (CAT). The hGSH required for ASC regeneration in the matrix originates in the cytosol. Bacteroids can also import hGSH from the cytosol but do not export glutathione (GSH). 
where (Gogorcena et al. 1997). Plants were in the vigorous vegetative growth stage and nodules were 3 weeks old when harvested.

\section{Purification of mitochondria and organelle markers.}

All steps were performed at 0 to $4^{\circ} \mathrm{C}$, with modifications of published protocols (Dalton et al. 1993; Sandalio et al. 1987; Struglics et al. 1993). Nodules (approximately $10 \mathrm{~g}$ ) were ground very gently in a mortar with $30 \mathrm{ml}$ of a medium con-

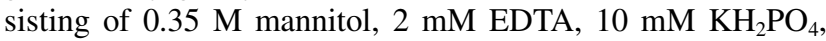
$2 \%(\mathrm{wt} / \mathrm{vol})$ polyvinylpolypyrrolidone, $0.4 \%$ (wt/vol) fatty acidfree bovine serum albumin (BSA), and $30 \mathrm{mM}$ morpholinepropanesulfonic acid (MOPS) ( $\mathrm{pH}$ 7.2). The extract was squeezed through four layers of cheesecloth and centrifuged twice at $4,000 \times g$ for $5 \mathrm{~min}$. The final supernatant was centrifuged at $12,000 \times g$ for $15 \mathrm{~min}$, and the pellet was resuspended in $25 \mathrm{ml}$ of washing medium consisting of $0.3 \mathrm{M}$ mannitol, $1 \mathrm{mM}$ EDTA, $0.2 \%$ BSA, and $20 \mathrm{mM}$ MOPS (pH 7.2). The pellet obtained after new centrifugation was resuspended in $1.8 \mathrm{ml}$ of washing medium and layered on top of a Percoll gradient composed of $7.1 \mathrm{ml}$ of $10 \%$ ( $\mathrm{vol} / \mathrm{vol}$ ), $7.1 \mathrm{ml}$ of $15 \%$, $12.4 \mathrm{ml}$ of $35 \%$, and $6.3 \mathrm{ml}$ of $50 \%$. Each Percoll solution contained $0.25 \mathrm{M}$ sucrose, $0.2 \% \mathrm{BSA}$, and $10 \mathrm{mM}$ MOPS (pH 7.2). The 10 and $15 \%$ layers were supplemented with $100 \mathrm{mM}$ propane-1,2-diol to increase hydrophobicity of the Percoll layers (Schwitzguebel and Siegenthaler 1984). The tube was centrifuged at $13,000 \times g$ for $35 \mathrm{~min}$ in a $70 \mathrm{Ti}$ rotor in a L8-70M ultracentrifuge (Beckman Coulter, Fullerton, CA, U.S.A.). Mitochondria were removed, washed twice, resuspended in $2 \mathrm{ml}$ of wash medium, and applied to a second gradient composed of $9 \mathrm{ml}$ of $30 \%$ Percoll, $0.3 \mathrm{M}$ mannitol, $0.2 \%$ BSA, and $10 \mathrm{mM}$ MOPS (pH 7.2), and $22 \mathrm{ml}$ of the same solution, replacing mannitol with sucrose. The tube was centrifuged at $23,500 \times g$ for $35 \mathrm{~min}$ with the same rotor and ultracentrifuge. Mitochondria were recovered, washed, and resuspended in 0.5 to $1.5 \mathrm{ml}$ of wash medium, without BSA.

Mitochondria purification was monitored with organelle protein markers: cytochrome $c$ oxidase and malate dehydrogenase (mitochondria), uricase and catalase (peroxisomes), alanine dehydrogenase (bacteroids), and leghemoglobin (cytosol). Enzyme activities were measured at $25^{\circ} \mathrm{C}$, within linear range. Alanine dehydrogenase was assayed in aliquots from the different purification steps after sonication for $4 \times 30 \mathrm{~s}$ in a melting ice bath. The other marker enzymes and leghemoglobin were assayed in aliquots made up to $0.05 \%$ of Triton $\mathrm{X}$ 100 and sonicated for $2 \times 5 \mathrm{~s}$. The activities of cytochrome $c$ oxidase, uricase, and alanine dehydrogenase were measured following the oxidation of ferrous cytochrome $c$ (Schnarrenberger et al. 1971), uric acid (Hanks et al. 1981), and NADH (Reibach et al. 1981), respectively. Catalase activity was determined following the decomposition of $\mathrm{H}_{2} \mathrm{O}_{2}$ at $240 \mathrm{~nm}$ (Aebi 1984). Leghemoglobin was quantified by the highly sensitive fluorometric assay of LaRue and Child (1979).

\section{Antioxidant enzymes and inhibitor studies.}

Peroxidases and SOD were extracted by resuspension of mitochondria in $20 \mathrm{mM}$ MOPS $(\mathrm{pH} \mathrm{7.2)}$ containing $0.05 \%$ Triton X-100 and $1 \mathrm{mM}$ EDTA and by subsequent sonication for $2 \times 5 \mathrm{~s}$ in an ice bath. The other antioxidant enzymes were extracted in the same medium supplemented with $2 \mathrm{mM} \beta$ mercaptoethanol, before sonication. APX activity was deter- mined following the oxidation of ascorbate at $290 \mathrm{~nm}$ (Nakano and Asada 1981), including 0.5 mM EDTA in the reaction mixture. GPX activity was assayed with $20 \mathrm{mM}$ pyrogallol as substrate, including $0.5 \mathrm{mM}$ pCMS in the reaction mixture (Amako et al. 1994). For inhibition studies of APX and GPX activities, mitochondrial extracts were preincubated in buffer for $8 \mathrm{~min}$ with the inhibitors and concentrations stated in Table 2. DR activity was assayed by the formation of ascorbate at $265 \mathrm{~nm}$ (Nakano and Asada 1981). MR and hGR activities were determined following the oxidation of NADH and NADPH, respectively (Gogorcena et al. 1997). SOD activity was measured following the inhibition of the superoxide radical-mediated reduction of ferric cytochrome $c$. One unit of SOD was defined as the amount of enzyme required to inhibit the reaction by $50 \%$ (McCord and Fridovich 1969). Isozymes of SOD were separated on $10 \%$ polyacrylamide mini-gels and differentiated by incubation with $3 \mathrm{mM} \mathrm{KCN}$ or $5 \mathrm{mM} \mathrm{H}_{2} \mathrm{O}_{2}$ (Becana et al. 1989). The reaction mixtures for all antioxidant enzyme assays contained a final concentration of 0.01 or $0.02 \%$ Triton X-100.

\section{Thiol tripeptides in the mitochondria, cytosol, and whole nodule extracts.}

Thiol tripeptides were extracted from mitochondria by incubation in $200 \mathrm{mM}$ methanesulfonic acid containing $0.5 \mathrm{mM}$ diethylenetriaminepentaacetic acid (DTPA) for $1 \mathrm{~h}$ at $0^{\circ} \mathrm{C}$ and then quantified as monobromobimane derivatives using HPLC with fluorescence detection (Matamoros et al. 1999).

To determine the thiol tripeptide composition of the cytosol and whole nodules, two samples of $50 \mathrm{mg}$ of nodules, harvested from the same plants, were extracted in parallel as follows. The first sample was extracted with $250 \mu \mathrm{l}$ of isosmotic medium (0.35 M mannitol; $30 \mathrm{mM}$ MOPS; and $0.5 \mathrm{mM}$ DTPA, pH 6.0) and centrifuged. The supernatant (cytosol) was mixed immediately with an equal volume of $400 \mathrm{mM}$ methanesulfonic acid containing $0.5 \mathrm{mM}$ DTPA and was cleared by centrifugation. The second sample of nodules was extracted with $500 \mu \mathrm{l}$ of acid medium (200 mM methanesulfonic acid and $0.5 \mathrm{mM}$ DTPA), and the extracts were sonicated and centrifuged. The supernatants of both extracts were used for thiol analysis, as reported for mitochondria.

\section{Organelle integrity and intramitochondrial localization of enzymes.}

The integrity of the outer and inner mitochondrial membranes was assessed by measuring the latency of cytochrome $c$ oxidase and malate dehydrogenase, respectively. Malate dehydrogenase activity was assayed following the oxidation of $\mathrm{NADH}$ at $340 \mathrm{~nm}$ in a medium consisting of $50 \mathrm{mM}$ potassium phosphate (pH 7.0), 0.02\% Triton X-100, $1 \mathrm{mM} \mathrm{KCN,} 1 \mathrm{mM}$ oxaloacetate, $5 \mu \mathrm{l}$ of extract, and $0.2 \mathrm{mM}$ NADH to initiate the reaction, in a final volume of $1 \mathrm{ml}$. Latency of enzymes was determined in hyposmotic medium (broken organelles) and isosmotic medium (intact organelles), with or without $0.02 \%$ Triton X-100, respectively, except in the case of cytochrome $c$ oxidase, in which a noninhibitory $0.01 \%$ Triton concentration was used. The latency, expressed in percent, was calculated by the formula of Burgess et al. (1985): latency $=100 \times[($ activity + Triton) - (activity - Triton)]/(activity + Triton)

For solubilization studies, mitochondria were resuspended in $1 \mathrm{ml}$ of $10 \mathrm{mM} N$-2-hydroxyethylpiperazine- $N^{\prime}$-2-ethane- 
sulfonic acid (pH 7.2), $10 \%$ glycerol, and 1 mM EDTA. After two freeze-thaw cycles in liquid nitrogen, the extract was centrifuged at $13,000 \times g$ for $10 \mathrm{~min}$ and the supernatant was centrifuged at $100,000 \times g$ for $45 \mathrm{~min}$. The pellet was resuspended in $1 \mathrm{ml}$ of the same buffer containing $0.01 \%$ Triton X-100, and the enzyme activities were measured in both fractions. Solubility of the enzymes was expressed as the percent of the total activity that is present in the soluble fraction.

Mitochondria were separated into outer membrane-enriched fraction, inner membrane-enriched fraction, and matrix. Intact mitochondria (showing latencies of cytochrome $c$ oxidase and malate dehydrogenase $>90 \%$ ) were subjected to controlled hyposmotic shock to preferentially disrupt the outer membrane. Mitochondria were resuspended in $5 \mathrm{mM}$ MOPS (pH 7.0) containing $8 \mathrm{mM}$ mannitol and incubated with occasional shaking at $0^{\circ} \mathrm{C}$ for $60 \mathrm{~min}$, by which time the latency of cytochrome $c$ oxidase had decreased to $50 \%$. The suspension, containing partially broken mitochondria and membranes, was loaded onto a two-phase sucrose gradient and the outer membrane-enriched fraction was obtained as described by Mannella (1987). Mitoplasts were collected in the pellet of the sucrose gradient and broken by osmotic shock in $5 \mathrm{mM}$ MOPS $(\mathrm{pH}$ 7.0) for 1 to $2 \mathrm{~h}$ with periodic shaking. The broken mitoplasts were centrifuged at $100,000 \times g$ for $45 \mathrm{~min}$ to produce the inner membrane-enriched fraction, whereas the supernatant was taken as the matrix fraction. The marker enzymes, antimycin A-insensitive cytochrome $c$ reductase (outer membrane), cytochrome $c$ oxidase (inner membrane), and malate dehydrogenase (matrix), were used to characterize the three fractions. The activities of cytochrome $c$ oxidase and malate dehydrogenase were assayed as described above, and antimycin Ainsensitive cytochrome $c$ reductase activity was assayed according to Mannella (1987).

\section{ACKNOWLEDGMENTS}

We thank G. Rodríguez for help with harvesting nodules and M. R. Clemente for help with analyzing thiols in cytosol and whole nodule extracts. D. A. Dalton thanks the NSF for partial funding support (grant no. IBN-950749). This work was fully supported by grant no. PB98-0522 from the Dirección General de Investigación Científica (Ministry of Science and Technology, Spain).

\section{LITERATURE CITED}

Aebi, H. 1984. Catalase in vitro. Methods Enzymol. 105:121-126.

Amako, K., Chen, G. X., and Asada, K. 1994. Separate assays specific for ascorbate peroxidase and guaiacol peroxidase and for the chloroplastic and cytosolic isozymes of ascorbate peroxidase in plants. Plant Cell Physiol. 35:497-504.

Becana, M., Paris, F. J., Sandalio, L. M., and Del Río, L. A. 1989. Isoenzymes of superoxide dismutase in nodules of Phaseolus vulgaris L., Pisum sativum L., and Vigna unguiculata (L.) Walp. Plant Physiol. 90:1286-1292.

Becana, M., Dalton, D. A., Moran, J. F., Iturbe-Ormaetxe, I., Matamoros, M. A., and Rubio, M. C. 2000. Reactive oxygen species and antioxidants in legume nodules. Physiol. Plant. 109:372-381.

Bergmann, L., and Rennenberg, H. 1993. Glutathione metabolism in plants. Pages 109-123 in: Sulfur Nutrition and Assimilation in Higher Plants. L. J. De Kok, ed. SPB Academic Publishing, The Hague, The Netherlands.

Bunkelmann, J. R., and Trelease, R. N. 1996. Ascorbate peroxidase: A prominent membrane protein in oilseed glyoxysomes. Plant Physiol. 110:589-598.

Burgess, N., Beakes, G. W., and Thomas, D. R. 1985. Separation of mi- tochondria from microbodies of Pisum sativum L. cv. Alaska cotyledons. Planta 166:151-155.

Carlenor, E., Persson, B., Glaser, E., Andersson, B., and Rydström, J. 1988. On the presence of a nicotinamide nucleotide transhydrogenase in mitochondria from potato tuber. Plant Physiol. 88:303-308.

Chen, G.-X., and Asada, K. 1989. Ascorbate peroxidase in tea leaves: Occurrence of two isozymes and the differences in their enzymatic and molecular properties. Plant Cell Physiol. 30:987-998.

Chen, G.-X., and Asada, K. 1990. Hydroxyurea and $p$-aminophenol are the suicide inhibitors of ascorbate peroxidase. J. Biol. Chem. 265:2775-2781.

Dalton, D. A. 1995. Antioxidant defenses of plants and fungi. Pages 298-355 in: Oxidative Stress and Antioxidant Defenses in Biology. S. Ahmad, ed. Chapman and Hall, New York.

Dalton, D. A., Baird, L. M., Langeberg, L., Taugher, C. Y., Anyan, W. R., Vance, C. P., and Sarath, G. 1993. Subcellular localization of oxygen defense enzymes in soybean (Glycine max [L.] Merr.) root nodules. Plant Physiol. 102:481-489.

De Leonardis, S., Dipierro, N., and Dipierro, S. 2000. Purification and characterization of an ascorbate peroxidase from potato tuber mitochondria. Plant Physiol. Biochem. 38:773-779.

Gogorcena, Y., Gordon, A. J., Escuredo, P. R., Minchin, F. R., Witty, J. F., Moran, J. F., and Becana, M. 1997. $\mathrm{N}_{2}$ fixation, carbon metabolism, and oxidative damage in nodules of dark-stressed common bean plants. Plant Physiol. 113:1193-1201.

Hanks, J. F., Tolbert, N. E., and Schubert, K. R. 1981. Localization of enzymes of ureide biosynthesis in peroxisomes and microsomes of nodules. Plant Physiol. 68:65-69.

Horemans, N., Foyer, C. H., Potters, G., and Asard, H. 2000. Ascorbate function and associated transport systems in plants. Plant Physiol. Biochem. 38: 531-540.

Ishikawa, T., Yoshimura, K., Sakai, K., Tamoi, M., Takeda, T., and Shigeoka, S. 1998. Molecular characterization and physiological role of a glyoxysome-bound ascorbate peroxidase from spinach. Plant Cell Physiol. 39:23-34.

Jiménez, A., Hernández, J. A., Del Río, L. A., and Sevilla, F. 1997. Evidence for the presence of the ascorbate-glutathione cycle in mitochondria and peroxisomes of pea leaves. Plant Physiol. 114:275-284.

Jiménez, A., Hernández, J. A., Ros Barceló, A., Sandalio, L. M., Del Río, L. A., and Sevilla, F. 1998. Mitochondrial and peroxisomal ascorbate peroxidase of pea leaves. Physiol. Plant. 104:687-692.

Klapheck, S. 1988. Homoglutathione: Isolation, quantification, and occurrence in legumes. Physiol. Plant. 74:727-732.

LaRue, T. A., and Child, J. J. 1979. Sensitive fluorometric assay for leghemoglobin. Anal. Biochem. 92:11-15.

Lash, L. H. 1995. Intracellular distribution of thiols and disulfides: Assay of mitochondrial glutathione transport. Methods Enzymol. 252: 14-25.

Mannella, C. A. 1987. Isolation of the outer membrane of plant mitochondria. Methods Enzymol. 148:453-464.

Matamoros, M. A., Moran, J. F., Iturbe-Ormaetxe, I., Rubio, M. C., and Becana, M. 1999. Glutathione and homoglutathione synthesis in legume root nodules. Plant Physiol. 121:879-888.

McCord, J. M., and Fridovich, I. 1969. Superoxide dismutase. An enzymic function for erythrocuprein (hemocuprein). J. Biol. Chem. 244: 6049-6055.

Millar, A. H., Atkin, O. K., Lambers, H., Wiskich, J. T., and Day, D. A. 1995a. A critique of the use of inhibitors to estimate partitioning of electrons between mitochondrial respiratory pathways in plants. Physiol. Plant. 95:523-532.

Millar, A. H., Day, D. A., and Bergersen, F. J. 1995b. Microaerobic respiration and oxidative phosphorylation by soybean nodule mitochondria: Implications for nitrogen fixation. Plant Cell Environ. 18:715726.

Mittler, R., and Zilinskas, B. A. 1991. Purification and characterization of pea cytosolic ascorbate peroxidase. Plant Physiol. 97:962-968.

Moran, J. F., Iturbe-Ormaetxe, I., Matamoros, M. A., Rubio, M. C., Clemente, M. R., Brewin, N. J., and Becana, M. 2000. Glutathione and homoglutathione synthetases of legume nodules. Cloning, expression, and subcellular localization. Plant Physiol. 124:1381-1392.

Nakano, Y., and Asada, K. 1981. Hydrogen peroxide is scavenged by ascorbate-specific peroxidase in spinach chloroplasts. Plant Cell Physiol. 22:867-880.

Õba, K., Ishikawa, S., Nishikawa, M., Mizuno, H., and Yamamoto, T. 
1995. Purification and characterization of L-galactono- $\gamma$-lactone dehydrogenase, a key enzyme for ascorbic acid biosynthesis, from sweet potato roots. J. Biochem. 117:120-124.

Prasad, T. K., Anderson, M. D., and Stewart, C.R. 1995. Localization and characterization of peroxidases in the mitochondria of chillingacclimated maize seedlings. Plant Physiol. 108:1597-1605.

Puppo, A., Dimitrijevic, L., and Rigaud, J. 1987. $\mathrm{O}_{2}$ consumption and superoxide dismutase content in purified mitochondria from soybean root nodules. Plant Sci. 50:3-11.

Rasmusson, A. G., and Møller, I. M. 1990. NADP-utilizing enzymes in the matrix of plant mitochondria. Plant Physiol. 94:1012-1018.

Reibach, P. H., Mask, P. L., and Streeter, J. G. 1981. A rapid one-step method for the isolation of bacteroids from root nodules of soybean plants, utilizing self-generating Percoll gradients. Can. J. Microbiol. 27:491-495.

Sandalio, L. M., Palma, J. M., and Del Río, L. A. 1987. Localization of manganese superoxide dismutase in peroxisomes isolated from Pisum sativum L. Plant Sci. 51:1-8.

Scandalios, J. G., Tong, W.-F., and Roupakias, D. G. 1980. Cat3, a third gene locus coding for a tissue-specific catalase in maize: Genetics, intracellular location, and some biochemical properties. Mol. Gen. Genet. 179:33-41.

Schnarrenberger, C. A., Oeser, A., and Tolbert, N. E. 1971. Development of microbodies in sunflower cotyledons and castor bean endosperm during germination. Plant Physiol. 48:566-574.

Schwitzguebel, J.-P, and Siegenthaler, P.-A. 1984. Purification of peroxisomes and mitochondria from spinach leaf by Percoll gradient centrifugation. Plant Physiol. 75:670-674.

Siendones, E., González-Reyes, J. A., Santos-Ocaña, C., Navas, P., and Córdoba, F. 1999. Biosynthesis of ascorbic acid in kidney bean: LGalactono- $\gamma$-lactone dehydrogenase is an intrinsic protein located at the mitochondrial inner membrane. Plant Physiol. 120:907-912.

Smith, J., and Shrift, A. 1979. Phylogenetic distribution of glutathione peroxidase. Comp. Biochem. Physiol. 63B:39-44.

Struglics, A., Fredlund, K. M., Rasmusson, A. G., and Møller, I. M. 1993. The presence of a short redox chain in the membrane of intact potato tuber peroxisomes and the association of malate dehydrogenase with the peroxisomal membrane. Physiol. Plant. 88:19-28. 